\title{
On the Configuration of the Magnetotail Near Midnight During Quiet and Weakly Disturbed Periods: Magnetic Field Modeling
}

\author{
H. I. WeSt, JR., AND R. M. BUCK \\ Lawrence Livermore Laboratory, University of California, Livermore, California 94550
}

\author{
M. G. Kivelson
}

Department of Earth and Space Sciences and Institute of Geophysics and Planetary Physics, University of California Los Angeles, California 90024

\begin{abstract}
Energetic-particle pitch angle data and vector magnetometer data, measured along the Ogo 5 inbound orbit near midnight on three magnetically quiet days, August 2,4 , and 25,1968 , were used to infer the magnetic tail field configuration for each day. For the first 2 days the particles, when initially detected, showed isotropic pitch angle distributions (PAD's) (indicative of nonadiabatic motions resulting from the breakdown of the guiding center invariants $\mu$ and $J$ ) but later made rapid transitions to the butterfly PAD (indicative of adiabatic guiding center motion). The lower-rigidity particles made the PAD transitions first and were followed in turn by the higher-rigidity particles as Ogo neared the earth. We have used a simple algebraic model which has allowed us to fit both the magnetic field and the pitch angle transition data. For the latter data fit, the detailed particle trajectories were followed along the model field lines to the neutral sheet. The particles were required to execute adiabatic guiding center motion if they were started from positions on the satellite orbit nearer earth than the point of observed changes in the PAD. They were required to undergo nonadiabatic motion at some part of their trajectory if they were started at a point on the satellite orb|t farther from the earth than the PAD transition point. The results are presented in terms of model coefficients for each day. The model fields are plotted, the cross-tail currents are derived, and particle motion is studied in the model fields. It was found that $79-\mathrm{keV}$ electrons were in the nonadiabatic mode when they were on field lines that crossed the neutral sheet beyond $11 R_{E}$ on August 2; on August 25, a less taillike day, this point was $17 \pm 1 R_{E}$. For $10-\mathrm{keV}$ protons the inferred points are 8.5 and $11.7 R_{E}$, respectively, for these 2 days. This is interesting because protons of about this energy carry a large part of the cross-tail current. It is expected that when data from particles of a wide range of rigidity are available, this method of inferring the tail field configuration should be very powerful.
\end{abstract}

\section{INTRODUCTION}

In the companion paper [West et al., 1978], hereafter called paper 1 , we presented energetic electron data acquired on inbound orbits of Ogo 5 near midnight during quiet periods. Here we provide field modeling of selected orbits to show quantitatively the differences that can exist for the quiet-day, near-earth tail configuration near midnight. We have chosen to model the data of August 2, 4, and 25 because they exemplify the total body of data. Also, these data were acquired fairly close to the noon-midnight meridian, thus simplifying the problem of transforming from the dual system of coordinates (dipole near the earth to GSM coordinates down the tail, coupled by an intervening hinging region) to coordinates symmetrical in $z$ relative to the neutral sheet.

For the August 25 data (September 17 and 18 data from paper 1 are similar) we found the butterfly pitch angle distribution (PAD) starting with the first detection of energetic electrons $(\sim 79 \mathrm{keV})$ at $\sim 15 R_{E}$ on the inbound orbit. Clearly, the electrons were on field lines that allow adiabatic guiding center motion. Conversely, for August 2 and 4 (exemplifying the rest of the body of data) the first detected electrons on the inbound orbit showed the isotropic PAD (indicative of nonadiabatic motion, i.e., the breakdown of first and second invariants $\mu$ and $J$ ), which later changed to the butterfly PAD (indicative of adiabatic guiding center motion) as Ogo 5 neared the earth. We interpret isotropic distributions as evidence that the elec-

This paper is not subject to U.S. copyright. Published in 1978 by the American Geophysical Union. trons are on field lines that thread the neutral sheet in a region of weak field and small field curvature so that the electrons cannot execute adiabatic motion. As the electrons scatter out of the neutral sheet region, they return to guiding center motion but still appear to be isotropic at the satellite (except for the loss cone, which we cannot observe). A more thorough discussion of the process is presented in the section on method in paper 1; further insight can be gained from the papers by West et al. [1973a, b] and West and Buck [1974] and from the results section of the present paper.

In this paper we have used a field model that has had good success in modeling both the magnetic field data obtained on the inbound orbit and the rigidity-dependent position at which the PAD changes occurred along the orbit. In adjusting the model to the PAD data we resorted to following the detailed trajectories of the particles from the point of the PAD changes in the orbit to where the model field lines cross the neutral sheet farther down the tail. The results in the plasma sheet are quite convincing and, we believe, still maintain some validity in the tail lobes. From the results of the modeling study we have studied particle motion in the magnetotail, have inferred the cross-tail current in the near-earth magnetotail, and have obtained information on the pressure balance between particles and fields. Most significantly, we have established a method of using particles as probes that with the proper instrumentation can be of extraordinary power in assessing the configuration of the magnetotail.

The particle data used in this paper came from the Lawrence Livermore Laboratory (LLL) scanning electron and proton 


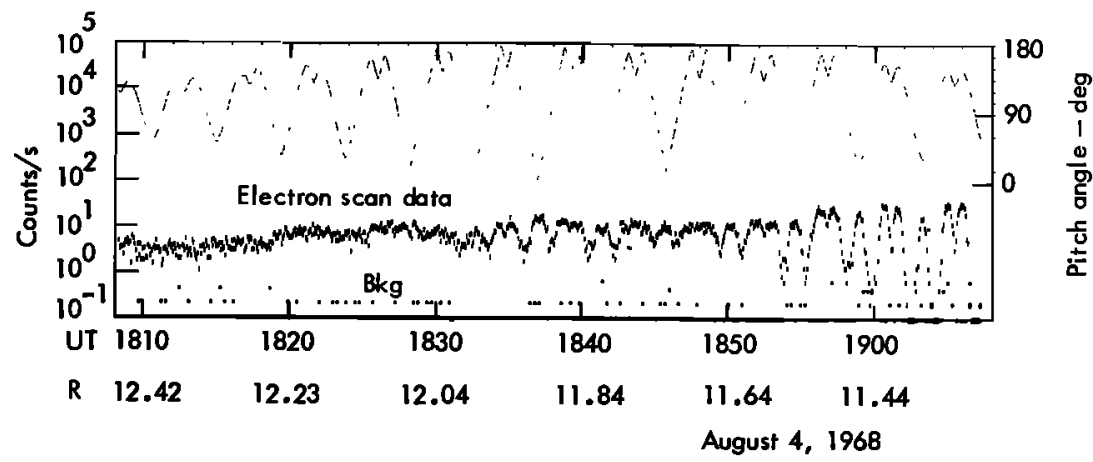

Fig. 1. PAD transition data of August 4, 1968, displayed on an expanded scale. The zigzag pattern at the top gives the instantaneous pitch angle, read off the scale on the right. Complementary data are presented in Figure 14 of paper 1.

spectrometers on Ogo 5. The instrument is described briefly in paper 1 and in more detail by West et al. [1969, 1973a]. The magnetic field data came from the UCLA triaxial fluxgate magnetometer experiment on Ogo 5 [Aubry et al., 1971], spot checks on the data being provided by the Goddard Space Flight Center (GSFC) data (B. Ledley, private communication, 1977) obtained on Ogo 5.

\section{DATA}

The energetic-electron data and associated magnetic field data used in our modeling study were introduced in paper 1 and are presented in Figures 1, 2, and 14 of that paper. The August 25 data in the bottom panel of Figure 1 of paper 1 show the scan-modulated counting rates. Almost from the first detection, except near $1120 \mathrm{UT}$, we find a high degree of scan modulation of the counting rates of these $79-\mathrm{keV}$ electrons. In the top panel of the figure we note that a neutral sheet crossing occurred between 1200 and 1300 UT.

Similar data for August 4, 1968, are shown in Figure 14 of paper 1 . There we note that the transition from isotropic to butterfly PAD occurred at about 1830 UT, and from the top panel we see that this is near the neutral sheet crossing. This interesting region of change in PAD is expanded in Figure 1 of the present paper. The zigzag pattern at the top shows the instantaneous look angle of the spectrometer with respect to B as the spectrometer scanned back and forth. From this figure we see that the butterfly PAD began to emerge at 1832 UT but was not complete until 1852 UT.

Data acquired on August 2, 1968, are shown in Figure 2 of paper 1 . The fluctuations prior to 0520 UT suggest plasma sheet motions. The satellite was dropping down from the north lobe of the tail, and thus the particle decrease suggests a southward motion of the plasma sheet. At about 0633 UT a transition from isotropy to the butterfly PAD occurs. This transition region is shown in more detail in Figure 2 of the present paper. In contrast to the August 4 data we find abrupt changes in the PAD only during a few minutes of the satellite travel ( $\sim 0.1 R_{E}$ on August 2, compared with $0.5 R_{E}$ on August 4).

PAD transition data for other channels of the electron spectrometer to $822 \mathrm{keV}$ also were obtained on August 2. Energetic protons were available on this orbit as well. For protons, there

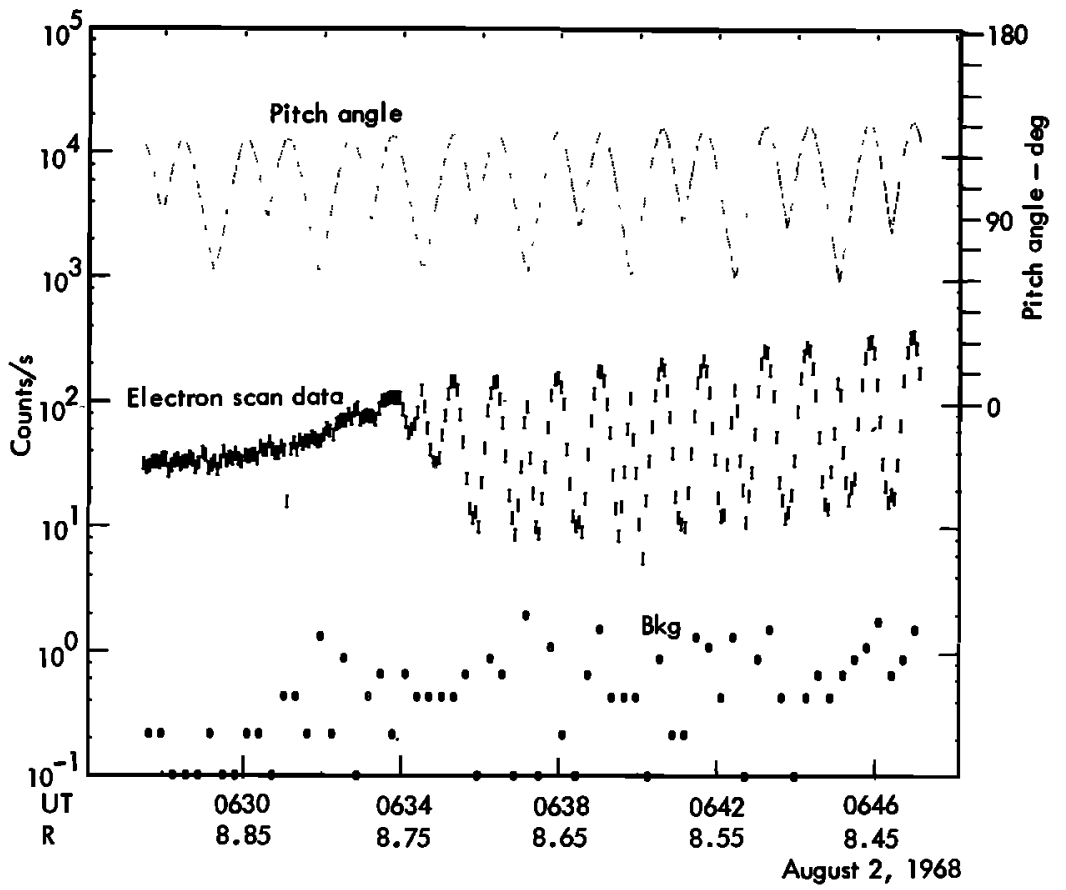

Fig. 2. Pitch angle changes for 79-keV electrons for the inbound orbit on August 2, 1968. The change from isotropy to butterfly PAD occurs from approximately 0633 to 0636 UT. The zigzag pattern at the top indicates the instantaneous pitch angle, read off the scale on the right. Complementary data are presented in Figure 2 of paper 1. 
was not only a transition from isotropy to butterfly distribution but also a strong east-west effect, typical of large spatial particle gradients [Buck et al., 1973], which resulted in marked scan-modulated counting rates in regions of both adiabatic guiding center and nonadiabatic motion. Careful considerations were necessary to establish the isotropy to butterfly transition points. The transition points for the five lowest energy electron channels and the two lowest energy proton channels are given in Table 1 . For the protons we have given the beginning and ending points of the region over which the PAD changes were occurring. Because of the subjectivity of these determinations an error of $\pm 0.1 \boldsymbol{R}_{E}$ is possible.

It is important that the magnetic field data used for the modeling be reasonably accurate; this is especially true of the $B_{Z \mathrm{GSM}}$ component, since it determines to a large extent where the field lines cross the neutral sheet. There were small offsets in the UCLA data (C. T. Russell, private communication, 1975). Russell applied corrections to the data based on the rubidium vapor magnetometer data obtained in the magnetosphere by the GSFC experiment of Heppner, Ledley, and Skillman, combined with interplanetary comparisons with Explorer 33 and 34 data. We have also cross-compared the UCLA data with the GSFC vector magnetometer data for August 2 and 4. Significant differences that would affect our modeling are not present. From the consistency of the data and discussions with the experimenters we believe that we know $B_{Z \mathrm{GSM}}$ on these 2 days to within at least $2 \gamma$.

\section{The Model AND Method}

We need a model that holds near midnight in the cusp region to at least $20 R_{E}$ down the tail. Various partial solutions exist. For example, Pudovkin and Tsyganenko [1973] have studied particle motions in a model that has some validity down the tail. However, in their model, $B_{z}$ goes to zero at $z=0$ and does not satisfy the requirement that $\nabla \cdot B=0$. Bird and Beard [1972] and Choe and Beard [1974] have done self-consistent calculations of the currents and fields in the magnetotail. In addition, D. B. Beard and D. J. Jackson (unpublished manuscript, 1975) have provided a recipe to connect the nearearth field lines to the magnetotail. The model that we finally adopted is essentially that of Beard and Jackson down the tail but is simplified in the near-earth regions. There have also been attempts to model the total magnetosphere [e.g., Olson and Pfizer, 1974a; Mead and Fairfield. 1975] that provide insight into the tail problem but are not specific enough for our needs. At the completion of the present work we became aware of the theoretically derived tail models of Toichi [1972] and
Kan [1973]. These models are interesting but would only partially meet our needs.

In selecting a model a question arises concerning the finite thickness of the neutral sheet region suggested by the analysis of the Imp 1 data by Speiser and Ness [1967]. They seemed to find a region well down the tail over which the field reversal occurs in the range 0.1-1 $R_{E}$. This prompted Pudovkin and Tsyganenko [1973] to include in their model a region of reduced-field spatial variation relative to that in the rest of the plasma sheet. The concept of a neutral sheet of finite thickness seems to be a confused issue in the literature. All too often, the term neutral sheet is used when plasma sheet is meant. Here we identify the whole plasma sheet as the region carrying the cross-tail current. However, in general, the current is expected to peak in the region of the neutral sheet which we consider to be a region of field reversal of zero thickness.

In providing a model of the plasma sheet it is necessary to remember that even on a quiet day, fairly large temporal variations can be occurring. Hopefully, one encounters fluctuations around a steady state value, so that these fluctuations cancel out in the modeling. Thus the model can only hope to provide the average of the data, not the fluctuations, unless the fluctuations are occurring on a time scale of several hours.

We have adopted a model of a divergenceless field, symmetrical in $z$, which contains a near-earth part to handle the region $\sim 6-10 R_{E}$ and a part containing the tail contribution to handle the region from 10 to $20 R_{E}$ and possibly farther. The model is

$$
\begin{aligned}
& B_{z}=\left(a_{1}-a_{6} z^{2}+a_{8} z^{4}\right) \exp \left(-a_{2} x\right)+\left(a_{3}-a_{5} \ln \cosh a_{7} z\right) x^{-a_{4}} \\
& B_{x}=-z \frac{\left(2 a_{6}-4 a_{8} z^{2}\right) \exp \left(-a_{2} x\right)}{a_{2}}-\frac{\left(a_{5} a_{7} \tanh a_{7} z\right) x^{-a_{4}+1}}{a_{4}-1}
\end{aligned}
$$

Here $x=\left|X_{\mathrm{GsM}^{2}}+Y_{\mathrm{GSM}^{2}}\right|^{1 / 2}, z$ is the height above the neutral sheet (restricted to a few earth radii), $B_{z} \approx B_{Z \mathrm{GSM}}$, and $B_{x} \approx$

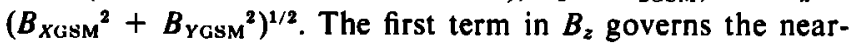
earth variations, and the second term governs the variations down the tail. $B_{x}$ and $B_{z}$ are related through the requirement that $\nabla \cdot \mathbf{B}=0$. Symmetry conditions obviously require an even variation in $z$ for $B_{z}$ and an odd variation for $B_{x}$.

For the near-earth part we might expect the first term in the dipole expansion $\left(1 / x^{3}\right)$ to be useful, but unfortunately, it does not fall off fast enough to describe the observed fields. However, a semilog plot of the $B_{z}$ data reveals a well-defined, exponentially dependent region of the data suggesting the exp $\left(-a_{2} x\right)$ term. For the variations down the tail $\left(x^{-a_{1}}\right)$ we used the compilation of experimental results given by Olson [1974],

\begin{tabular}{|c|c|c|c|c|c|c|c|}
\hline Energy, keV & Time, UT & $\boldsymbol{R}, \boldsymbol{R}_{E}$ & $\theta_{\mathrm{GSM}}, \mathrm{deg}$ & $\lambda_{m}, \operatorname{deg}$ & $\phi_{\mathrm{GSM}}$, deg & $x_{0}^{*}$ & $z_{0}^{*}$ \\
\hline \multicolumn{8}{|c|}{ Electrons } \\
\hline 79 & 0632.8 & 8.785 & 14.4 & 7.4 & 199.7 & 8.51 & 1.45 \\
\hline 158 & 0634.0 & 8.755 & 14.3 & 7.3 & 199.8 & 8.48 & 1.43 \\
\hline 266 & 0635.8 & 8.709 & 14.2 & 7.2 & 199.9 & 8.44 & 1.40 \\
\hline $478 \dagger$ & 0642.5 & 8.538 & 14.0 & 6.8 & 200.4 & 8.28 & 1.30 \\
\hline $822 \dagger$ & 0646.7 & 8.430 & 13.8 & 6.5 & 200.7 & 8.19 & 1.25 \\
\hline \multicolumn{8}{|c|}{ Protons } \\
\hline $100-150$ & 0706-0728 & $7.92-7.31$ & $12.8-11.6$ & $5.2-3.5$ & $202.0-204.0$ & $7.72-7.16$ & $0.98-0.66$ \\
\hline $230-570$ & 0714-0754 & $7.70-6.55$ & $12.4-9.7$ & $4.6-1.1$ & $202.7-206.8$ & & \\
\hline
\end{tabular}

TABLE 1. Isotropic to Butterfly Transitions for August 2 Data

*The transition points for pitch angle changes in terms of the neutral sheet coordinates; $x=\left|X_{\mathrm{GsM}}{ }^{2}+Y_{\mathrm{GsM}}\right|^{2 / 2}$, and $z$ is the height above the predicted neutral-center sheet positions in earth radii.

† More accurately, this is the first clear appearance of these electrons rather than a clearly defined transition in PAD. 
from which we determined $a_{4}$ to be $\sim 1.7$. In so doing we had hoped to take advantage of Olson's critical examination of the experimental literature. Of course, $a_{4}$ can be expected to vary from day to day, but, even worse, it is largely based on lobe field data, and we have no way of knowing that $a_{4}$ is independent of $z$. In light of this we note that Bird and Beard [1972], through their self-consistent calculations, obtain 1.3 for $a_{4}$. In any case, this difference in $a_{4}$ values does not result in much of an error over most of the region in which we are working. Because errors are corrected by other terms, the $a_{4}$ value has little effect on the data fit. The differences, however, do produce uncertainties in attempting to extrapolate the results down the tail.

One may include a constant of integration term $\left(-z a_{0}\right)$ in the $B_{x}$ equation, but the other terms were adequate without this. The $a_{\theta}$ term was important in the near-earth region. The $a_{8}$ term is important in the near-earth region when it is desirable to go to large $z(\gtrsim 2)$. The $a_{3}$ and $a_{5}$ terms in the tail part represent the $z$ variations in the plasma sheet. The $\tanh a_{7} z$ term, of course, provides the desired roll-off of the field at the edge of the plasma sheet to the constant lobe values down the tail. The term was derived theoretically by Harris [1962] and has been used by other workers. We have also had some success with a power series in the $z$ variation, that is, the second part of $B_{x}$ equaling (power series in $z$ ) $x^{-a_{4}+1}$. However, with that model, one has to go to special effort to provide rolloff of $B_{x}$ at the edge of the plasma sheet. Ideally, we would prefer a term in $B_{x}$ of the form tanh $\left(a_{7} z+a_{9} z^{3}\right)$, but it is not readily integrable as would be necessary in providing the corresponding $B_{z}$ term.

An important problem in this study was the estimation of the height of Ogo 5 above the neutral sheet, especially in the hinging region. The Russell-Brody formula [Russell and Brody, 1967] was inadequate for our purposes, but it did serve as a guide to developing a more adequate solution. The assumptions that we made agree with the conclusions of Thomas and Hedgecock [1975] regarding the near-earth tail field, Fortunately, the August 4 and 25 data contain neutral sheet crossings. Our procedure was to determine the angle between the projections of the satellite radius vector on the dipole equatorial plane and on the GSM equatorial plane and then, starting at $\left|X_{\mathrm{GSM}}\right|=5 R_{E}$, to taper the dipole equatorial plane into the neutral sheet at $11 R_{E}$ measured radially. It may be expected that the GSM elevation angle of the $11-R_{E}$ vector to the neutral sheet, $\alpha$, and the dipole plane elevation angle $\beta$ are related by a constant ratio. For seven case studies within $\pm 1 \mathrm{~h}$ from midnight, $\alpha / \beta=0.68 \pm 0.06$. When data containing neutral sheet crossings are used, the predictions with this model in the hinging region and nearby magnetotail were generally within $0.1 R_{E}$ of measurement. At greater than $1 \mathrm{~h}$ from midnight a fair fit was obtained by reducing the three parameters $\left(\alpha, \beta\right.$, and the $11-R_{E}$ vector) by $\cos \phi_{\mathrm{Gs}}$, but more work is needed to refine the procedure. In any case, the neutral sheet position thus obtained was close to the extension of the dipole plane. In addition, the data fit was sensitive to the neutral sheet determination. With minor adjustments in $\alpha$ we believe that the neutral sheet for the August 2 data was known to $\lesssim 0.1 R_{E}$ in the region of major interest $\left(6-12 R_{E}\right)$.

For all of the August data we had to make use of data obtained well into the hinging region. Here we found it necessary to transform $B_{X G S M}$ and $B_{Z G S M}$ into new coordinates, symmetrical in $z$. This was accomplished by expressing $B_{x}$ as the component tangent to the curved neutral sheets and $B_{z}$ as the component perpendicular. With this transformation, quite an improvement in the modeling was noted for smaller values of $x\left(7-9 R_{E}\right)$.

In general, in considering particle motion in the magnetotail, one should include the cross-tail electric field [Speiser, $1965,1967,1971]$. Since conditions were magnetically quiet, we considered it acceptable first to ignore the effects of the cross-tail electric field in terms of particle energization and convection. Later in the paper we examine convection, along with some of the details of the motion during breakdown of the invariants; this suggests that convection was not particularly important.

\section{Fits to the Model and Trajectory Studies}

For inputs to the models we, of course, used the field observations along the satellite orbit in the plasma sheet. We also required that $B_{x}$ roll off properly to the lobe value at the edge of the plasma sheet and that the cross-tail current derived from $(1 / \mu) \nabla \times B$ roll off to zero at the edge. Finally, the rigiditydependent pitch angle transition data should be predicted by the model.

The fits to the field data were fairly direct once the model and the procedure were established. Because of the short-term fluctuations in the data, there may be some question about the model $z$ dependence in the region of the neutral sheet crossing. However, we feel that $B_{x} \sim \tanh a_{7} z$ works rather well, although the use of $\tanh \left(a_{7} z+a_{9} z^{3}\right)$ would improve the fit somewhat for the August 4 data. The fit coefficients are listed in Table 2, and the field lines are plotted in Figure 3.

The fit of the August 2 data went especially well. Once the coefficients were established by using the observed field values, we were able to reproduce the pitch angle changes without resorting to changes in the coefficients. Here the choice between the hyberbolic function model and the power law (in $z$ ) becomes clear; the adopted model immediately provides the right field variations near the edge of the plasma sheet needed to reproduce the observed pitch angle changes.

Table 1 gives the neutral sheet coordinates $\left(x_{0}, z_{0}\right)$ of the pitch angle changes (isotropy to butterfly PAD) along the inbound orbit for the August 2 data. We require that the field model allow a particle to move from the point of observed PAD changes along the field line to a region in the model neutral sheet at which the guiding center invariants just start to break down in terms of increasing $x_{\text {ns }}$ (the value of $x$ in the neutral sheet). (Note that strictly speaking, the particle motion down the tail should not be called nonadiabatic, since both Speiser [1970] and Sonnerup [1971] have shown that new invariants are applicable there. Here, however, we shall use the term nonadiabatic to mean violation of the first and second adiabatic invariants $\mu$ and $J$.) To identify the dividing line in the neutral sheet between the two classes of particle motion, we can use the Alfvén criterion [Alfvén and Fälthammar, 1963],

TABLE 2. Field Modeling Fit Coefficients

\begin{tabular}{cccc}
\hline & August 2 & August 4 & August 25 \\
\hline$a_{1}$ & 5300 & 8500 & 13,000 \\
$a_{2}$ & 0.658 & 0.65 & 0.654 \\
$a_{3}$ & 210 & 123 & 450 \\
$a_{4}$ & 1.7 & 1.7 & 1.7 \\
$a_{5}$ & 95 & 134 & 131 \\
$a_{6}$ & 550 & 700 & 1,000 \\
$a_{7}$ & 1.1 & 0.9 & 0.54 \\
$a_{8}$ & 0 & 0 & 0.10 \\
\hline
\end{tabular}




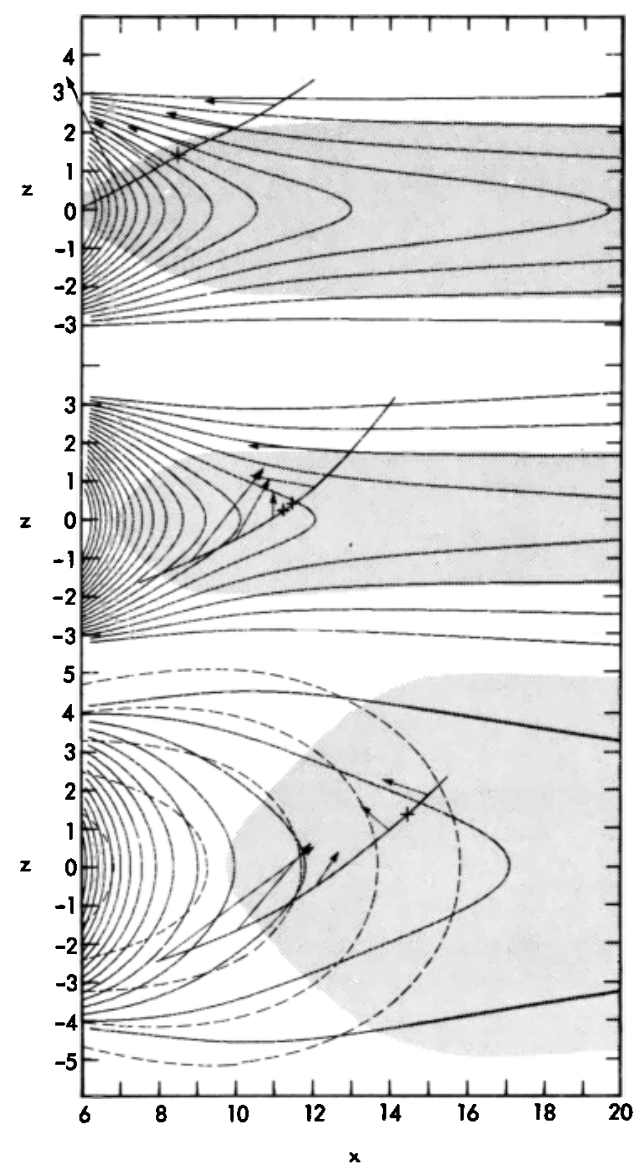

Fig. 3. Results of modeling the field configuration for August 2,4, and 25,1968 . The results are shown for the expected region of validity of the model. Field lines are drawn for intervals of constant flux. Field vectors are sketched along the orbit in each case to indicate the success of the modeling. The field vectors for August 2 are drawn to one-half the scale of those for the other 2 days. The pluses along the trajectories indicate the point of transition from isotropy to butterfly PAD's for 79-keV electrons. The shaded area represents the plasma sheet; the inward terminus was determined by evaluating the current, and the edge of the shaded region down the tail represents the transition to zero current and open field lines. The dashed field lines for August 25 are from the Olson and Pfitzer [1974a] model.

which states that for adiabatic guiding center motion the perturbation expansion parameter $R_{c} / \rho$ should be $\gtrsim 10$; here $\rho$ is the radius of curvature of the particle trajectory, and $R_{c}$ is the curvature of the field line. In the neutral sheet we find $\nabla_{1} B=$ $\partial B_{x} / \partial z=B_{z} / R_{c}[$ Roederer, 1970]. Whence in the neutral sheet we have

$$
k \equiv R_{c} / \rho=\left[B_{\mathrm{zns}}^{2}\left(\partial B_{x} / \partial z\right)_{\mathrm{ns}}\right]\left(1 / B \rho_{\perp}\right)
$$

where we can expect $k$ to be of the order of $10 . B \rho_{\perp}$ is a constant of motion, the rigidity, which for $E_{1-s}(79,158,266$, 478 , and $822 \mathrm{keV}$ ) is $0.154,0.226,0.306,0.443$, and $0.645 \gamma R_{E}$, respectively. We now have a curvature equation as a function of $x_{n s}$ that can be iterated to give the neutral sheet crossing.

In principle, the $k$ equation provides a quick check on the model parameters without having to go through the procedure of following the detailed trajectories of the particles. However, as a check on the procedure we have followed the particles by computer. We started particles off at the $x_{0}$ values listed in Table 1 at increments above and below the $z_{0}$ values. Determining the position of invariant breakdown can be somewhat subjective; one does not observe obvious changes in the tra- jectory plots (some are shown and discussed in the next section). Hence the accurate determination of $\mu$ and $J$ is important. In any case, the determination of the transition point $\left(x_{n s}\right)$ for the electrons in the neutral sheet was not overly subjective, having a variation of only $\pm 0.1 R_{\Sigma}$. In the case of the protons, there was more subjectivity. The $\mu$ and $J$ calculations were not quite as accurate (showed slight fluctuations in regions where adiabaticity was expected), and there seems to be evidence of phase variations. The value of $k$ obtained for $E_{1}$ is $9.8 \pm{ }_{1.0}^{2.4}$, which is typical for the electrons. The average $k$ for $E_{1}-E_{5}$ is $10.2 \pm{ }_{1.0}^{1.2}$. These values are extraordinarily close to the Alfven value. For the 100 - to $150-\mathrm{keV}$ protons we obtained $6.8 \pm 1.5$; the value of $k=10$ predicts PAD transitions outside our error but not greatly so. In the case of the proton trajectory studies we noted that the protons were approaching the neutral sheet with flatter pitches than the electrons (because of the difference in starting points), suggestive of a pitch angle dependence. More detailed study shows the pitch angle effects to be negligible.

The results of our trajectory analysis for the August 2 pitch angle transition data are shown in Figure 4 . The open circles show the results of following the field line passing through the PAD transition points $\left(x_{0}, z_{0}\right)$ (Table 1$)$ into the model neutral sheet. The solid circles show the results of the trajectory study of particles starting from the same $x_{0}$ value but with the $z_{0}$ value picked to identify the field line of the PAD changes in the model through the trajectory analysis. The results appear to be quite satisfactory.

The results of the trajectory calculation were then applied to the August 4 data. The scan-modulated fluxes are shown in Figure 1, their instantaneous pitch angles (the zigzag pattern) being given to the right of the figure. The magnetic field data

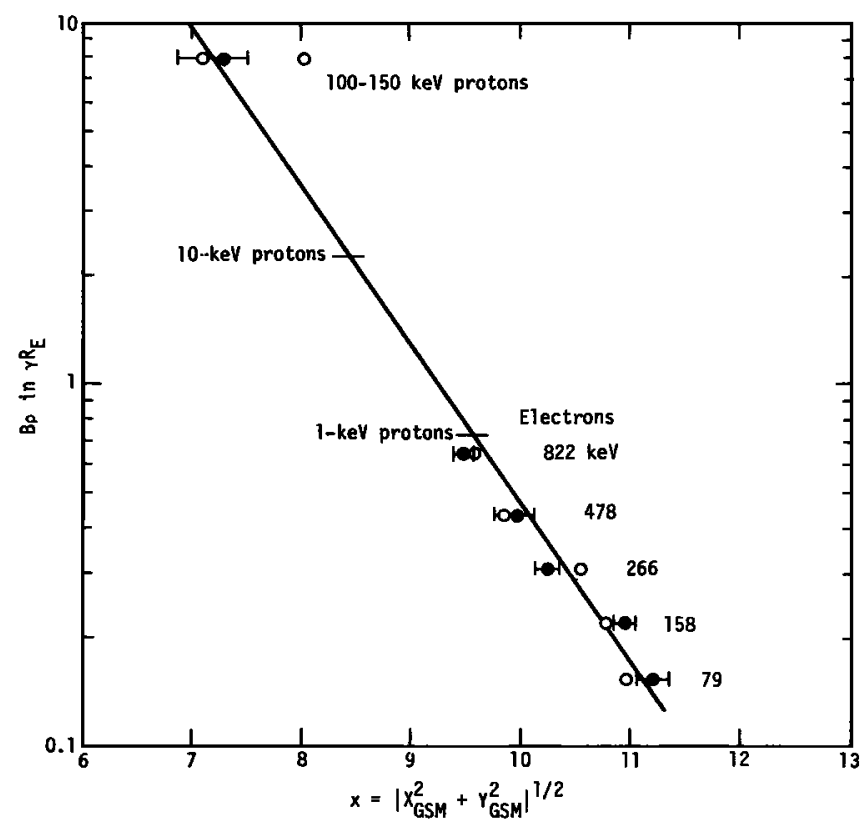

Fig. 4. Dividing line in the neutral sheet between adiabatic and nonadiabatic motion for the August 2 modeling study. The abscissa is the neutral sheet position, and the ordinate is the particle rigidity. The open circles are the result of tracing the field lines from the observed PAD changes $\left(x_{0}, z_{0}\right)$, along the model field lines, to the neutral sheet. The solid circles are from the trajectory study, and the error bars indicate possible uncertainties in the calculation. The points at which 1- and 10-keV protons are expected to make their transition are indicated, as based on their rigidity. 
are given in the top panel of Figure 14 of paper 1. The neutral sheet crossing, as determined from fitting the data to the model, was at $1912 \mathrm{UT}$, and the transition from isotropy to butterfly $\left(x_{0}, z_{0}\right)$ was equal to $(11.44,0.46)$ and $(11.20,0.23)$, respectively, which, followed into the neutral sheet, give crossings of 11.92-11.33 $R_{E}$. These values are in good agreement with the $k$ equation, which predicts $11.5 R_{E}$. It is interesting that the transition to the butterfly distribution covered such a wide region. The finite channel width of the electron spectrometer (triangular response, $60-100 \mathrm{keV}$ ) could not be responsible for this. Computationally, we find little pitch angle dependency. To a small extent we find that the transition region reflects the phases of the particle motion, that is, that some avenues of approach to the high-curvature region allow easier passage without scatter than do others. Also, we expect that the wide transition region reflects temporal variations in the magnetic field as Ogo traversed this region. However, we believe that a study of the details of the particle motion largely explains the results; we defer this discussion until later.

\section{RESULTS}

\section{Field Configuration}

In Figure 3 we provide field line plots for the August 2, 4, and 25 data. To help assess the accuracy of the results, we have sketched the satellite orbit and have drawn averaged field vectors along the orbit (note that the field vectors for August 2 are drawn to one-half the scale used for the other days). Also, along the orbits we have indicated the points of the pitch angle changes for 79-keV electrons for the August 5 and 4 data and for the first appearance of electrons for the August 25 data. It is unfortunate that we do not have transition points earlier in the orbit in each case to help establish the field geometry near the edge of the plasma sheet and farther down the tail. This would have required an instrument able to measure particles of lower rigidity than could be measured on Ogo 5 .

On the August 25 plot of Figure 3 we show the results of the Olson and Pfitzer [1974a] model. We note that even for the most dipolelike configuration in our study of the quiet magnetotail, our results are more taillike than the Olson-Pfitzer model. We expect that their model is more applicable in the tail regions away from midnight.

The August 2 results agree quite well with the August 4 results, but the August 25, extraquiet-day results show marked differences from the others, the most obvious difference being the relatively dipolelike configuration. Also, the August 2 and 4 data show field lines bending slightly away from the plasma sheet (as a result of the action of the $a_{5}$ coefficient causing $B_{z}$ to become negative). Such bending also occurs for August 25 but is off the edge of the plot. This bending away is interpreted as the transition from closed to open field lines.

The results in Figure 4 showing the transition points in the neutral sheet for $\mu-J$ violation, we believe, provide convincing proof of the validity of our modeling efforts. The most obvious immediate conclusion is that electron scattering (as a result of field configuration) operates on all-energy electrons near the edge of the plasma sheet, but then to an observer in the auroral zone the precipitated electron spectrum hardens equatorward. In Figure 4 we have shown the pitch angle transition points for 1- and 10-keV protons, since protons of these energies carry an appreciable part of the cross-tail current [e.g., Hones et al., 1976]. The transition points are 9.6 and $8.5 R_{E}$, and for August 25 they are 13.1 and $10.9 \boldsymbol{R}_{E}$.

\section{Particle Trajectories}

Once the field model coefficients are established, we can make meaningful studies of the particle trajectories. Primarily, we use the August 2 results. In Figures 5 and 6 we show some plots generated in studying the 79-keV electron PAD changes. In each case the electron was started off at $x=8.50$ but at different values of $z$; particle motion is shown in the region of the neutral sheet. For $z=1.45$ in Figures $5 a-5 c$ the motion is barely adiabatic in the region of the neutral sheet. Even so, we see the effect of the high-curvature region such that motion in the $y$ direction is greatly enhanced. For $z=1.75$ in Figures $5 d-$ $5 f$ the region of $\mu-J$ violation in the vicinity of the neutral sheet is fairly large; only when approximately $0.2 R_{E}$ or more from the neutral sheet do we find approximate guiding center motion.

Figure 6 shows electrons entering the looping mode in the vicinity of the neutral sheet. For these curves, especially the $z$ $=2.05$ curves (Figures $6 d-6 f), B_{z}$ is weak enough that the motions in the $x-y$ and $y-z$ planes are largely decoupled; we see the motion about $B_{z}$ in Figures $6 c$ and $6 e$.

Figure 7 shows particle motion farther down the tail. Figures $7 a-7 c$ show $79-\mathrm{keV}$ electrons under the influence of a -2 $\mathrm{mV} / \mathrm{m}$ dawn to dusk cross-tail field. Inward motion can be seen in Figure $7 a$ following the first bounce in the plasma sheet. Figure $7 c$ shows the cross-tail motion. Here we see the results of, first, westward gradient $B$ drift (a result of the negative field gradient away from the neutral sheet), then looping the neutral sheet eastward, then westward gradient $B$ drift (this time farther from the neutral sheet), then looping the neutral sheet eastward again, and finally, the return to guiding center motion near the edge of the plasma sheet.

Figure $7 d-7 f$ show the motion of 822-keV electrons injected at $30 R_{E}$ down the tail. Again we note (Figure 7e) the strong decoupling between the motion in the $x-y$ and $y-z$ planes. Figure $7 f$ shows the marked effects of the alternation between looping the neutral sheet eastward and westward gradient $B$ drifting.

The results of both Figure 6 and Figure 7 show strong evidence, at times, of little or no drift in the eastward direction. One wonders about the importance of such effects in magnetospheric dynamics. Indeed, Stern and Palmadesso [1975] have shown for a specific model $\left(B_{z}=\right.$ const, $B_{x}$ is an odd function of $z$ ) that nondrift orbits can result. (By this we mean that for electrons the effects of gradient $B$ drifting westward just cancel the eastward curvature drift operating most strongly in the vicinity of the neutral sheet.) In our present studies we have found times when the cross-tail motion has seemed to stagnate, and this may occur for as much as $1 \mathrm{~s}$ of motion $\left(23-R_{E}\right.$ travel for $79-\mathrm{keV}$ electrons). Invariably, however, the particle (we have studied electrons at 79 and $822 \mathrm{keV}$ and protons at 100 and $10 \mathrm{keV}$ ), as it returns to guiding center motion, ends up with a net motion eastward for electrons or westward for protons as a result of strong curvature drift in the region of the neutral sheet encounter. Particle motion was also studied in the nonlooping modes. Here, although there is no momentary trapping in the neutral sheet region, the azimuthal drift rate is less than that for the region of the looping mode. For $79-\mathrm{keV}$ electrons, all starting at $x=8.5$, the drift rate for $z=1.45$ and $P A=120^{\circ}$ is $0.045 R_{E} / \mathrm{s}$; for $z=1.75$ and $P A=120^{\circ}$ it is $0.068 R_{E} / \mathrm{s}$; and for $z=2.05$ and $P A=120^{\circ}$ (enters the looping mode) it is $0.075 R_{E} / \mathrm{s}$. The rates are quite model dependent. For a model with $B_{x}$ linear in $z$ down the tail $\left(B_{z}=\right.$ 
$\left.\left(a_{3}-a_{5} z^{2}\right) x^{-a_{4}}, B_{x}=-\left[z a_{6} /\left(a_{4}-1\right)\right] x^{-a_{4}+1}\right)$ the drift rates are about 3 times as fast as those above. This model, in agreement with our earlier results, did not produce nondrift orbits, although for periods up to $1 \mathrm{~s}$ it appeared to do so. We, of course, found nondrift motion with the model used by Stern and Palmadesso [1975], a model which may describe a limited region of the magnetotail under special conditions.

In Figures $7 a-7 c$ we have shown weak effects of convective electric fields. Indeed, we have studied the effects of westward electric fields of -1 to $-3 \mathrm{mV} / \mathrm{m}$ and found results much the same as those reported before [Speiser, 1965, 1967; Sonnerup. 1971]. However, implicit in prior statements but not necessarily obvious is the fact that in the absence of neutral sheet scattering, the inward convection rate at, for example, the equator is independent of the pitch angle.

At this point the PAD transition regions for the $79-\mathrm{keV}$ electrons for the August 2 and 4 data discussed earlier can be more completely explained. As was pointed out earlier, the transition region for the August 4 data (Figure 1) maps to 0.6 $R_{E}$ in the neutral sheet (i.e., along $x$ ). Not so obvious is the fact that the relatively narrow transition region for the August 2 data (Figure 2) maps to $\sim 0.4 R_{E}$ in the neutral sheet. We note that in the region of the PAD changes, the motion (to first order), although violating $\mu-J$, still appears to be guiding center motion. For August 4 we injected $79-\mathrm{keV}$ electrons at $(x, z)=$ $(11.44,0.46)$ at pitch angles of $90^{\circ}$ and $150^{\circ}$ at 0 and $-3 \mathrm{mV} /$ m. The equatorial convection rate is $0.024 R_{E} / \mathrm{s} \mathrm{mV} / \mathrm{m}$ for both pitch angles of injection. From the work of Gurnett [1972] we find that the average cross-tail electric field is -0.2 $\mathrm{mV} / \mathrm{m}$. Whence a field of $-0.1 \mathrm{mV} / \mathrm{m}$ seems reasonable for August 4 , giving a convection rate of $\approx 0.0024 R_{E} / \mathrm{s}$.

The equatorial, azimuthal drift rates are $0.0714 R_{E} / \mathrm{s}$ for the
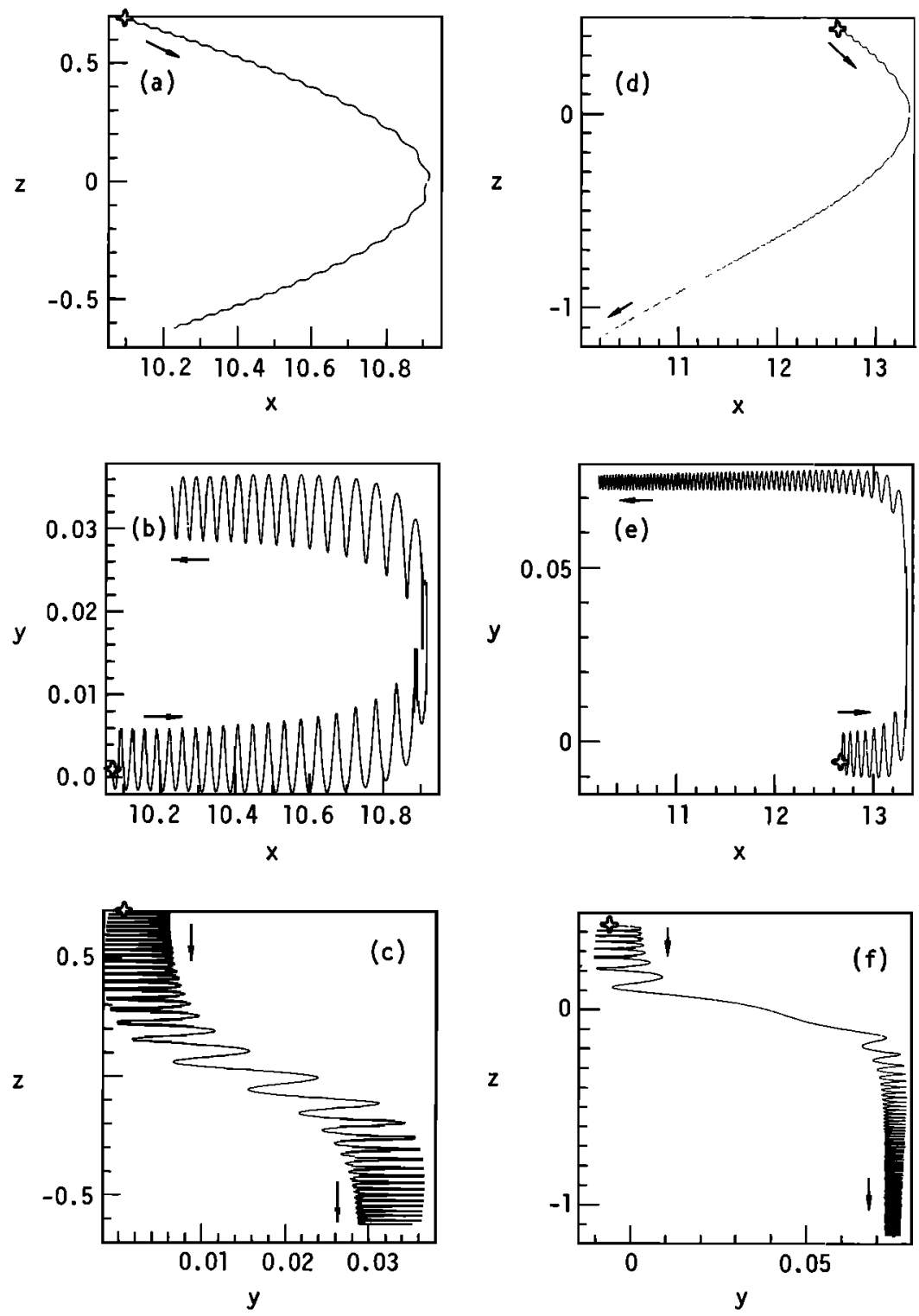

Fig. 5. Motion of 79-keV electrons showing guiding center motion in the vicinity of the neutral sheet for the August 2 model. $(a, b, c)$ The electron was injected at $x=8.5, z=1.45$, and $P A=130^{\circ}$. The motion is essentially adiabatic over its total trajectory. $(d, e, f)$ The electron was injected at $x=8.5, z=1.75$, and $P A=107^{\circ}$. Here the motion in the vicinity of the neutral sheet is distinctly nonadiabatic. (Note that here and in the text, $x$ and $y$ are positive down the tail and toward dusk, respectively.) 
$90^{\circ}$ electrons and $0.058 R_{E} / \mathrm{s}$ for the $150^{\circ}$ electrons. We expect that the boundary of $\mu-J$ violation has its most eastward extent near local midnight. For the August 4 data we note for each electron bounce in the region just tailward of the onset of $\mu-J$ violation that the pitch angle change per bounce (a few tenths of a second) is just a few degrees. The time for the scatter to $90^{\circ}$ is about $10 \mathrm{~s}$, with pitch angle dependent azimuthal drift and a small amount of convection occurring all the while. Near the earthward edge of the region the faster drift rate of the approximately $90^{\circ}$ particles tends to maintain the appearance of the butterfly PAD. We have only to go approximately $0.5 R_{E}$ down the tail to find that although the motion is to first appearances still guiding center, the pitch angle scatter per bounce is large enough to dominate all other effects. For the August 2 data, less scatter is needed in the neutral sheet to give the appearance of isotropy because of the location of the observation point.

\section{Cross-Tail Current}

We can gain more insight into our results by examining the cross-tail current through the evaluation of $(1 / \mu) \nabla \times \mathbf{B}$. We have not attempted to model the effects of the ring current, so in each case we have an inward terminus to the current system described by the model. The current, derived from the model, peaks in the region of the neutral sheet and rolls off to zero at the edge of the plasma sheet. Note that as we go down the tail, the width of the plasma sheet becomes constant and varies as $\left(\cosh ^{2} a_{7} z\right)^{-1} x^{-a_{4}+1}$. For the sheet to narrow with increasing $x$ we need $a_{7}$ to increase with $x$. Such a refinement is not warranted by the data, and because of this we evaluate the current to only $x=20$. The results are given in Table 3 .

From Table 3 we note that for both August 2 and August 4 the inward terminus of the current system is fairly close to the earth (5.6 and $6.8 R_{E}$, respectively), in contrast to the August
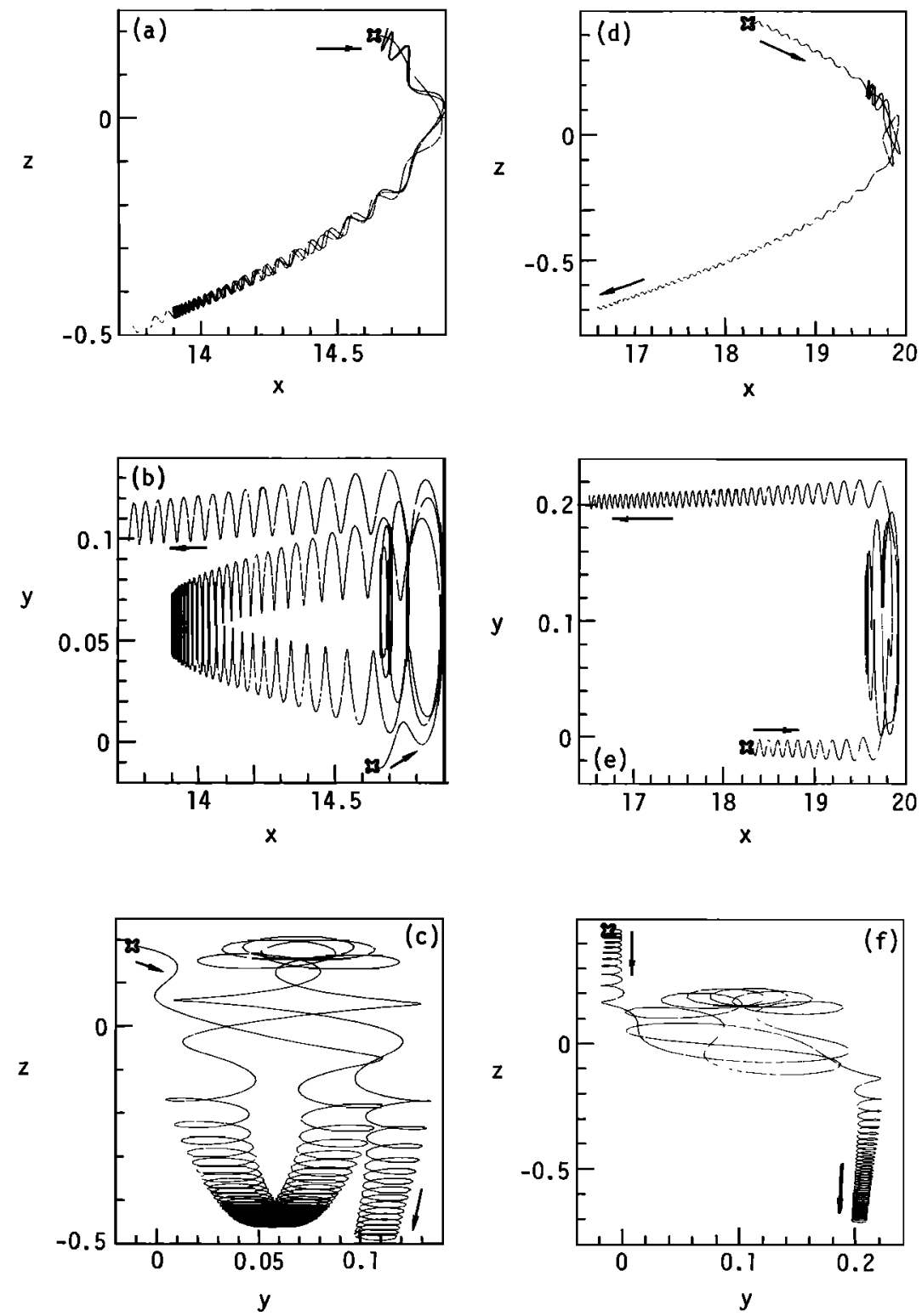

Fig. 6. Motion of 79-keV electrons showing $\mu-J$ violation in the vicinity of the neutral sheet for the August 2 model. (a. $b, c)$ The electron was injected at $x=8.5, z=1.85$, and $P A=107^{\circ}$. $(d, e, f)$ The electron was injected at $x=8.5, z=2.05$, and $P A=107^{\circ}$. Results for $x=8.5, z=1.95$, and $P A=107^{\circ}$ are shown in Figure 3 of paper 1 . 

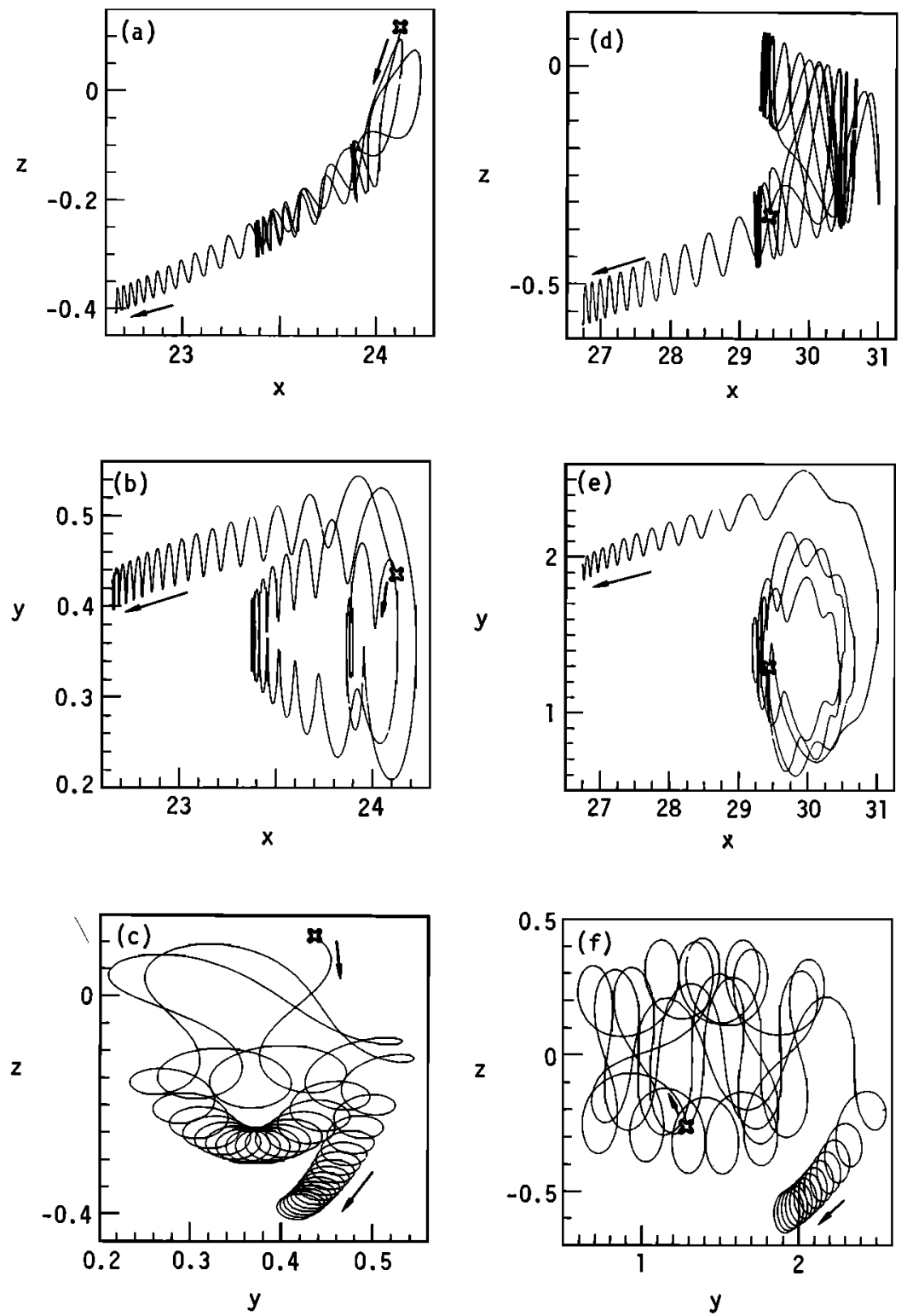

Fig. 7. Electron motion well down the tail using the August 2 model. $\left(a, b, c_{0}\right) E_{\mathrm{e}}=79 \mathrm{keV}, x=25, z=0.2, P A=95^{\circ}$, $E=-2 \mathrm{mV} / \mathrm{m}$, and the time period after injection is $3.60-3.99 \mathrm{~s}$. $(d, e, f) E_{\mathrm{e}}=822 \mathrm{keV}, x=30, z=0.2, P A=110^{\circ}$, and the time period after injection is $0.933-1.76 \mathrm{~s}$.

25 data, for which the current terminates at $9.7 R_{E}$. The details of the inner extent of the current system are shown in Figure 3. The peaks of the current systems are at 10 and $12 R_{E}$ for August 2 and 4, respectively, but the peak is at $15 R_{E}$ for August 25 . At this position we give the peak current density (per $R_{E}{ }^{2}$ ) and the current density integrated over the cross section of the plasma sheet (per $R_{E}$ ). For comparison, note that the peak current density from the Olson-Pfitzer model [Olson and Pfitzer, 1974a] is $0.1 \mathrm{MA} / \boldsymbol{R}_{E}{ }^{2}$ at $9.5 \boldsymbol{R}_{E}$. Finally, we give the cross-tail current evaluated to $20 R_{E}$; we obtain 2.90 , 2.92, and 1.18 MA for August 2, 4, and 25, respectively. As is expected, the August 25 data show a much weaker current system, consistent with the more dipolar field configuration on that day.

\section{Pressure Balance in the Magnetotail}

Since we have modeled the field configuration of the nearearth magnetotail, we should be able to say something about the forces balancing the magnetic tension. The pressure bal- ance problem in the plasma sheet has been discussed in some detail by Rich et al. [1972], Cole and Schindler [1972], and Pudovkin and Tsyganenko [1973]. Rich et al. state that 'the stress resulting from magnetic tension on the neutral sheet must, in a steady state, be balanced by one or a combination of (1) a pressure gradient in the direction along the axis of the tail, (2) a similar gradient of plasma-flow kinetic energy, and

TABLE 3. Estimates of the Cross-Tail Current in the Cusp Region to $20 R_{E}$

\begin{tabular}{lccc}
\hline & August 2 & August 4 & August 25 \\
\hline $\begin{array}{l}\text { Inward equatorial current } \\
\text { terminus, } R_{E}\end{array}$ & 5.6 & 6.8 & 9.7 \\
Peak in current, MA $/ \boldsymbol{R}_{E}^{2}$ & 0.150 & 0.131 & 0.037 \\
Cross-section current at $x$, & 0.260 & 0.274 & 0.122 \\
MA $/ R_{E}$ & & & \\
Value of $x, R_{E}$ & 10 & 12 & 15 \\
Current to $20 R_{E}$, MA & 2.90 & 2.92 & 1.18 \\
\hline
\end{tabular}


(3) the tension resulting from a pressure anisotropy, with $\boldsymbol{P}_{\|}>$ $P_{\perp}$ within the plasma sheet.' Rich et al. show that for case 1 above to hold true, $B$ in the neutral sheet must be $0.1 \gamma$; for case 2 to hold true the thickness of the neutral sheet must be several earth radii; and for case 3 to hold true the total pressure, electron plus proton, within the plasma sheet must be anisotropic and satisfy approximately the fire hose condition. From our work, case 3 is indicated, for which Rich et al. find $P_{\|} / P_{\perp}=\left(B_{T}^{2}+B^{2}\right) /\left(B_{T}{ }^{2}-B^{2}\right)$. Here $B_{T}$ is the lobe field just outside the plasma sheet, and $B$ is the field in the plasma sheet but away from the neutral sheet.

It appears that at $15 R_{E}$ down the tail this equation is applicable, and thus near the neutral sheet (taken as zero thickness) we have $P_{||} / P_{\perp}=1.02$ (August 2), 1.008 (August 4), and 1.23 (August 25). At $z=1 R_{E}$ we have $P_{\|} / P_{\perp}=4.45$ (August 2), 2.96 (August 4), and 1.85 (August 25). For the taillike case we find the need for a pressure anisotropy only as we move away from the neutral sheet, whereas for the more dipolelike case, some pressure anisotropy is needed throughout the plasma sheet. The August 25 data (Figure 1 of paper 1) show a very high pitch angle anisotropy for $\sim 79-\mathrm{keV}$ electrons; of course, it is not these electrons but the particles of much lower energy that are responsible for the pressure balance. It is interesting to note that the anisotropy must extend down to low energies. However, because we see very little diamagnetic effect in the magnetic field data, we expect that on this day, at $<15 R_{E}$, the low-energy particle population density was much lower than usual.

\section{Conclusions}

We have shown that magnetic field data can be supplemented with PAD data for energetic particles to infer the field configuration. These particles serve as probes of the field at some distance down the tail from the point of observation and provide definite information at the times of the changes in PAD. The procedure is potentially very powerful. With detectors capable of measuring particles over a wide range of rigidity we can probe the magnetotail over tens of earth radii, possibly even into the distant neutral point. Although there may be a problem with turbulence near the neutral sheet affecting the trajectories (especially those of lower energy), we expect that this approach can be useful during disturbed times as well as during quiet times.

Our studies show that even during the quietest period observed (August 25, 1968), the field configuration near midnight becomes taillike beyond $15 R_{E}$. This is contrary to the OlsonPfitzer model [Olson and Pfitzer, 1974a], which predicts field lines that are fairly dipolelike in these regions.

Olson and Pfitzer [1974b] show adiabatic trajectories of 5$\mathrm{keV}$ protons at $20 \boldsymbol{R}_{E}$ down the tail as calculated from their model. Although such trajectories may prevail well away from midnight, we have found that $10-\mathrm{keV}$ protons can undergo non-guiding center motion as close in as $8.5 \boldsymbol{R}_{E}$. Even with our most dipolelike data on August 25, we found that $10-\mathrm{keV}$ protons were no longer undergoing adiabatic motion beyond about $11.7 R_{E}$. The existence of particles in the region of $\mu-J$ violation results in a slow loss of particles to the atmosphere. Although the field configuration is the major contributor to this precipitation, turbulence in the vicinity of the neutral sheet is important too. The effects of turbulence are twofold: the first is the result of resonant interactions, with the resultant energy changes, and the second is the result of scattering as a result of configuration change, as described in this study.
Acknowledgments. This work was begun while one of us (H. I. W.) was a guest of the Institute of Geophysics and Planetary Physics at UCLA. The work at UCLA was supported by NASA under contract NGL 05-007-004. The work at LLL was conducted under the auspices of the U.S. Energy Research and Development Administration under contract W-7405-Eng-48. We thank P. Coleman and C. T. Russell for use of their magnetometer data from Ogo 5. We thank D. Hei (National Space Science Data Center) and B. Ledley for providing data from the GSFC magnetometer experiment. We thank S. W. H. Cowley for pointing out a small error to us.

\section{REFERENCES}

Alfvén, H., and C. G. Fälthammar, Cosmical Electrodynamics, Fundamental Principles, 2nd ed., Clarendon, Oxford, 1963.

Aubry, M. D., M. G. Kivelson, and C. T. Russell, Motion and structure of the magnetosphere, J. Geophys. Res., 76, 1673, 1971.

Bird, M. K., and D. B. Beard, The self-consistent geomagnetic tail under static conditions, Planet. Space Sci., 20, 2057, 1972.

Buck, R. M., H. I. West, Jr., and R. G. D'Arcy, Jr., Satellite studies of magnetospheric substorms on August 15, 1968: 7, Ogo 5 energetic proton observations-Spatial boundaries, J. Geophys. Res., 78, $3101,1973$.

Choe, J. Y., and D. B. Beard, The near earth magnetic field of the magnetotail current, Planet. Space Sci., 22, 609, 1974.

Cole, G. H. A., and K. Schindler, On the equilibrium configuration of the geomagnetic tail, Cosmic Electrodynamics, 3, 275, 1972.

Gurnett, D. A., Injun 5 observations of magnetospheric electric field and plasma convection, in Earth's Magnetospheric Processes, edited by B. M. McCormac, p. 233, D. Reidel, Hingham, Mass., 1972.

Harris, E. G., On a plasma sheet separating regions of oppositely directed magnetic field, Nuovo Cimento, 23, 115, 1962.

Hones, E. W., Jr., S. J. Bame, and J. R. Asbridge, Proton flow measurements in the magnetotail plasma sheet made with Imp 6,J. Geophys. Res., 81, 227, 1976.

Kan, J. R., On the structure of the magnetotail current sheet, $J$. Geophys. Res., 78, 3773, 1973.

Mead, G. D., and D. H. Fairfield, A quantitative magnetospheric model derived from spacecraft magnetometer data, $J$. Geophys. Res., 80, 523, 1975.

Olson, W. P., A model of the distributed magnetospheric currents, J. Geophys. Res., 79. 3731, 1974.

Olson, W. P., and K. A. Pfitzer, A quantitative model of the magnetospheric magnetic field, J. Geophys. Res., 79, 3739, $1974 a$.

Olson, W. P., and K. A. Pfitzer, Magnetospheric boundaries and fields, in Correlated Interplanetary and Magnetospheric Observations, edited by D. E. Page, D. Reidel, Hingham, Mass., $1974 b$.

Pudovkin, M. A., and N. A. Tsyganenko, Particle motion and currents in the neutral sheet of the magnetospheric tail, Planet. Space Sci., 21, 2027, 1973.

Rich, F. J., V. M. Vasyliunas, and R. A. Wolf, On the balance of stresses in the plasma sheet, J. Geophys. Res., 77, 4670, 1972.

Roederer, J. G., Dynamics of geomagnetically trapped radiation, in Physics and Chemistry in Space, vol. 2, Springer, New York, 1970.

Russell, C. T., and K. I. Brody, Some remarks on the position and shape of the neutral sheet, J. Geophys. Res., 72, 6104, 1967.

Sonnerup, B. U. O., Adiabatic particle orbits in a magnetic null sheet, J. Geophys. Res., 76, 8211, 1971 .

Speiser, T. W., Particle trajectories in model current sheets, 1, Analytical solutions, J. Geophys. Res., 70, 4219, 1965.

Speiser, T. W., Particle trajectories in model current sheets, 2, Applications to auroras using a geomagnetic tail model, J. Geophys. Res., 72, 3919, 1967.

Speiser, T. W., Conductivity without collisions or noise, Planet. Space Sci., 18, 613, 1970.

Speiser, T. W., The Dungey model of the magnetosphere and astrogeophysical current sheets, Radio Sci., 6, 315, 1971.

Speiser, T. W., and N. F. Ness, The neutral sheet in the geomagnetic tail: Its motion, equivalent currents, and field line connection through it, J. Geophys. Res., 72, 131, 1967.

Stern, D. P., and P. Palmadesso, Drift-free magnetic geometries in adiabatic motion, J. Geophys. Res., 80, 4244, 1975.

Thomas, B. T., and P. Hedgecock, Substorm effects in the neutral sheet inside $10 R_{E}$, in Magnetospheres of the Earth and Jupiter, edited by B. Formisano, p. 55, D. Reidel, Hingham, Mass., 1975.

Toichi, T., Two-dimensional equilibrium solution of the plasma sheet and its application to the problem of the tail magnetosphere, Cosmic Electrodynamics, 3, 81, 1972. 
West, H. I., Jr., and R. M. Buck, Pitch angle distributions of energetic electrons in the equatorial regions of the outer magnetosphereOgo 5 observations, in Magnetospheric Physics, edited by B. M. McCormac, p. 93, D. Reidel, Hingham, Mass., 1974.

West, H. I., Jr., J. H. Wujeck, J. H. McQuaid, N. C. Jenson, R. G. D'Arcy, Jr., R. W. Hill, and R. M. Bogdanowicz, The LRL electron and proton spectrometer on NASA's Orbiting Geophysical Observatory 5(E): Instrumentation and calibration, Rep. UCRL-50572, Lawrence Livermore Lab., Livermore, Calif., 1969.

West, H. I., Jr., R. M. Buck, and J. R. Walton, Electron pitch angle distributions throughout the magnetosphere as observed on Ogo 5 , J. Geophys. Res., 78, 1064, 1973 a.
West, H. I., Jr., R. M. Buck, and J. R. Walton, Satellite studies of magnetospheric substorms on A ugust 15, 1968: 6, Ogo 5 energetic electron observations-Pitch angle distributions in the nighttime magnetosphere, J. Geophys. Res., 78, 3093, 1973 b.

West, H. I., Jr., R. M. Buck, and M. G. Kivelson, On the configuration of the magnetotail near midnight during quiet and weakly disturbed periods: State of the magnetosphere, J. Geophys. Res., 83, this issue, 1978 .

(Received June 30, 1976; accepted December 21, 1977.) 